\title{
J-aggregate structure in a chloroform solvate of a 2,3-dicyanopyrazine dye -Separation of two-dimensional stacking dye layers by solvate formation-
}

Shinya Matsumoto, a* Emi Horiguchi-Babamoto, ${ }^{\mathrm{b}}$ Ryohei Eto, ${ }^{\mathrm{a}}$ Saori Sato, ${ }^{\mathrm{a}}$ Takashi Kobayashi, Hiroyoshi Naito, ${ }^{\mathrm{c}}$ Motoo Shiro ${ }^{\mathrm{d}}$ and Hiromi Takahashi ${ }^{\mathrm{e}}$

a Department of Environmental Sciences, Graduate School of Environment and Information Sciences, Yokohama National University, 79-2 Tokiwadai, Hodogaya-ku, Yokohama 240-8501, Japan. Fax: +81 45 339 3345; Tel: +81 45339 3366; E-mail: smatsu@edhs.ynu.ac.jp

b Department of Pharmaceutical Sciences, Faculty of Pharmaceutics, Musashino University, 1-1-20 Shinmachi, Nishitokyo-shi, Tokyo 202-8585, Japan

${ }^{\text {c }}$ Department of Physics and Electronics, Graduate School of Engineering, Osaka Prefecture University, 1-1 Gakuen-cho, Nakaku, Sakai, Osaka 599-8531, Japan

d X-ray Research Laboratory, Rigaku Corporation, 3-9-12, Matsubaracho, Akishima-shi, Tokyo 196-8666, Japan

e System Instruments Co., Ltd., 776-2 Komiyamachi, Hachioji-shi, Tokyo 192-0031, Japan

\begin{abstract}
Here, we report the J-aggregate structure of a chloroform solvate of 5-t-butyl-2,3-dicyano-6-[4-(dimethylamino)styryl]-pyrazine with a strong intramolecular charge transfer system. The dye was found to form a two-dimensional brick-wall structure separated by chloroform molecules in the solvated crystals, which exhibited intense red fluorescence. The absorption maximum of the solvate was also found to show a bathochromic spectral shift. These observed optical characteristics were interpreted in terms of excitonic intermolecular interaction based on two-dimensional brick-wall structure which is one of the proposed structures for J-aggregates.
\end{abstract}

Keywords: J-aggregates; pyrazine dye; chloroform solvate; red fluorescence; exciton interaction

${ }^{*}$ Corresponding author. Tel.: +81 45 339 3366; Fax: +81 $45 \quad 339$ 3345. E-mail address: 
smatsu@edhs.ynu.ac.jp (S. Matsumoto).

\section{Subdivision - numbered sections}

1. Introduction

2. Experimental section

2.1. Materials and equipments

2.2. Synthesis of 5-t-butyl-2,3-dicyano-6-[4-(dialkylamino)styryl]pyrazine

2.3. X-ray structural analysis

3. Results and discussion

4. Conclusions

Acknowledgements

References

\section{Introduction}

J-aggregates are one of the well-known supramolecular systems of organic dyes. ${ }^{[1]}$ They exhibit an intense, sharp absorption band with resonance fluorescence in the longer wavelength region compared with those of a component dye monomer. These particular spectral features may be ascribed to a resonance quantum effect known as exciton interaction within the aggregates. ${ }^{[2]} \mathrm{A}$ representative example of their practical applications is as a photosensitizer for colour photographic films. ${ }^{[1]}$ This industrial opportunity stimulated research into J-aggregates. One of the main research interests in J-aggregates is the structure. The structure of J-aggregates was first proposed with respect to the surface adsorption of J-aggregates on ionic substrates. ${ }^{[3]}$ With much experimental evidence of the formation of J-aggregates on the surface of ionic substrates, ${ }^{[1]}$ the structure of J-aggregates was further considered on the basis of theoretical treatments. ${ }^{[2]}$ There are also several reports on photographic images of J-aggregates using microscopic techniques. ${ }^{[4]}$ Comprehensive analyses of the crystal structure of cyanine dyes showed that many cyanine dyes crystallize in a $\pi-\pi$ stacking structure and the proposed one- or two-dimensional molecular arrangement of 
J-aggregates is recognized as a part of their three-dimensional crystal structures ${ }^{[5]}$ However, the detailed structure and dimensionality of J-aggregates are still unknown except for the $\mathrm{X}$-ray structure of a J-aggregate LB-film of a merocyanine dye reported by Kato et al. ${ }^{[6]}$ The size of J-aggregates is also controversial: a range of some molecules to some thousands molecules have been reported as the number of component molecules within one J-aggregate. ${ }^{[1(\mathrm{e}), 7]}$ An alternate opinion is that J-aggregates are a macroscopic molecular assembly composed of meso-scale molecular assemblies. ${ }^{[8]}$ The reported analysis of the J-aggregated LB-film ${ }^{[6]}$ demonstrated that a two-dimensional infinite dye network is also a form of J-aggregates, whilst that film may possess structural defects like mosaicity in single crystals.

In spite of the lack of clarity of the structure of J-aggregates, their spectral features have attracted much attention in state-of-the-art applications of organic dyes such as optical materials, ${ }^{[7(c), 7(d), 9]}$ organic solar cells, ${ }^{[10]}$ and organic light-emitting devices. ${ }^{[4(e),}{ }^{11]}$ Stable J-aggregates in solid films ${ }^{[12]}$ or in powdered form are required for these applications. It is also very important that these bulk J-aggregates can be prepared through a conventional method. To achieve these demands, J-aggregate formation in a stable crystalline state ${ }^{[13]}$ is a promising solution even though there is currently no design strategy for obtaining J-aggregate crystals. Here, we report the crystal structure and solid state optical properties of a chloroform solvate of a 2,3-dicyanopyrazine dye as an example of a potential crystal structure towards the design of J-aggregate crystals.

\section{Experimental section}

\subsection{Materials and equipments}

4-(Dimethylamino)benzaldehyde was purchased from Hayashi Pure Chemical Industries Ltd. 5-t-Butyl-2,3-dicyano-6-methylpyrazine ${ }^{[14]}$ was prepared as described in the literature.

Melting points were measured using a Yanaco MP-500P micro-melting-point apparatus. NMR spectra were obtained by a Jeol JNM-ECX-400 spectrometer. EIMS spectra were recorded on a Jeol JMS-GC mateII spectrometer. IR spectra were recorded on a Jasco FT-IR-4100 system for samples in a KBr pellet form. The elemental analysis was performed with a Elementar Vario EL III elemental analyzer. UV-vis 
absorption and fluorescence spectra in solution were taken on a Shimadzu UV3100PC UV/vis spectrometer and a Shimadzu RF5301PC fluorescence spectrometer, respectively. Solid-state absorption spectra were measured using a System Instruments SIS-50 surface and interface spectrometer based on optical waveguide spectrometry. Fluorescence quantum efficiency in the solid-state is determined using a blue diode laser (CrystaLaser), an integrating sphere (Labsphere), and photo-multichannel analyzer (Hamamatsu, PMA-11). After measured a reflected laser beam from a quarts substrate, we measured a reflected laser beam and fluorescence from the crystals placed on the substrate. Then, we calculated photon numbers of the fluorescence and the adsorbed laser beam. Fluorescence quantum efficiency was obtained as a ratio between the two photon numbers. ${ }^{[15]}$ Electronic states of this dye in the solvate were estimated by a semi-empirical molecular orbital calculation. The ground state dipole moment and the transition dipole moment were calculated by using the AM1 and the INDO/S Hamiltonians, respectively, on the basis of the crystal structure. These calculations were performed with a CAChe 5.2 program package. ${ }^{[16]}$

\subsection{Synthesis of 5-t-butyl-2,3-dicyano-6-[4-(dialkylamino)styryl]pyrazine}

To a solution of 5-t-butyl-2,3-dicyano-6-methylpyrazine (2.00 g, $10 \mathrm{mmol})$ in benzene (50 mL) was added piperidine (0.3 mL, $3 \mathrm{mmol})$ and 4-(dimethylamino)benzaldehyde (1.49 g, $10 \mathrm{mmol})$. The mixture was refluxed for $37 \mathrm{~h}$. The reaction mixture was cooled to room temperature and then poured into a $2 \% \mathrm{HCl}$ solution. The precipitate was filtered off and the filtrate was extracted with EtOAc three times. The combined organic layers were washed with brine and dried over $\mathrm{Na}_{2} \mathrm{SO}_{4}$. After removing the solvent, the residue was isolated by silica gel column chromatography (benzene) to give a solid product. This solid and the precipitate obtained by filtration were mixed and further purified by recrystallization from chloroform to obtain the target compound (1.56 g, 31\%) as reddish brown crystals. Yield 31\%; mp 263-264 ${ }^{\circ} \mathrm{C}$; Anal. Calcd for $\mathrm{C}_{20} \mathrm{H}_{21} \mathrm{~N}_{5}$ : C, 72.48; H, 6.39; N, 21.13\%. Found: C, 71.92; H, 6.28; N, 20.97\%; IR (KBr)/cm ${ }^{-1}$ 2225 (CN); ${ }^{1} \mathrm{H}$ NMR $\left(\mathrm{CDCl}_{3}\right) \delta 1.54$ (s, 9H), 3.10 (s, 6H), $6.74(\mathrm{~d}, J=8.0 \mathrm{~Hz}, 2 \mathrm{H}), 7.29(\mathrm{~d}, J=16.0 \mathrm{~Hz}$, 1H), $7.55(\mathrm{~d}, J=8.0 \mathrm{~Hz}, 2 \mathrm{H}), 8.03(\mathrm{~d}, J=16.0 \mathrm{~Hz}, 1 \mathrm{H}) ;{ }^{13} \mathrm{C} \mathrm{NMR}\left(\mathrm{CDCl}_{3}\right) \delta \quad 29.4,39.3,40.2,112.1$, 113.8, 114.3, 116.3, 123.4, 126.0, 130.1, 130.3, 143.0, 152.1, 152.9, 162.8; MS (EI) m/z $331\left(\mathrm{M}^{+}\right)$. 


\subsection{X-ray structural analysis}

Data collection of the solvate crystal was performed by a Rigaku RAXIS RAPID imaging plate diffractometer with graphite monochromated $\mathrm{Cu}-\mathrm{K} \alpha$ radiation. The structure was solved by direct method (SIR $2002^{[17]}$ ) and refined by full-matrix least-squares calculations. The non-hydrogen atoms were refined anisotropically. All hydrogen atoms were simply located at the calculated positions and their parameters were constrained. All calculations were performed using the CrystalStructure 3.8 crystallographic software package. ${ }^{[18]}$ Crystal data: $\mathrm{C}_{20} \mathrm{H}_{21} \mathrm{~N}_{5} \cdot \mathrm{CHCl}_{3}, M=450.80$, orthorhombic, $a=6.7574(5) \AA, b=11.1520(6) \AA$, $c=14.9279(9) \AA, V=1124.9(1) \AA^{3}, T=223.1 \mathrm{~K}$, space group $P m n 2_{1}, Z=2,11891$ reflections measured, 2202 independent reflections $\left(R_{\text {int }}=0.101\right)$. The final $R_{1}$ value $(I>2 \sigma(I))$, the final $w R\left(F^{2}\right)$ value (all data), and the GOF was $0.075,0.2163$ and 0.900 , respectively. The Flack parameter was $-0.05(4)$. The CCDC deposition number is CCDC834637.

\section{Results and discussion}

Dye 1, 5-t-butyl-2,3-dicyano-6-[4-(dimethylamino)styryl]-pyrazine, shown in Figure 1 was synthesized as one of a series of compounds to study the effect of the amino substituent on thin film growth by vacuum deposition. ${ }^{[19]}$ This dye system is also known to exhibit good fluorescence properties in the crystalline state. ${ }^{[14,20]}$ We carried out single crystal growth of $\mathbf{1}$ using liquid-liquid diffusion from chloroform and c-hexane. Lustrous red platelets were observed in the glass tube within a few days, and, after that, the red crystals transitioned into black block crystals and/or reddish purple flakes in the next several days. The red crystals were found to emit brightly under a UV lamp (Figure 2). They are stable at about $263 \mathrm{~K}$ without any colour change.

[Figure 1]

[Figure 2]

We initially thought that $\mathbf{1}$ had three crystal polymorphs; however, the red one was proved to be a chloroform solvate. Thermogravimetric (TG) analysis revealed that the red crystals include one chloroform 
molecule per dye (Figure 3). The other two crystals were identified as polymorphic forms without any solvent molecules and their structures and optical properties will be reported elsewhere.

[Figure 3]

X-ray structural analysis of the solvated crystals was carried out at $223 \mathrm{~K}$. The analytical data indicated that the structural unit is composed of one dye molecule and one chloroform molecule, which confirms the results of the TG analysis. The molecules are oriented on a mirror plane parallel to the $b c$ plane. This indicates that the molecules have a planar conformation. Figure 4 illustrates the crystal structure of the solvate. The crystal structure is characterized by a two-dimensional stacking dye layer and a layer composed of chloroform molecules. These two layers alternate along the $b$-axis to form the three-dimensional crystal structure. Figure 4 also shows a portion of the two-dimensional dye layer viewed from both the $a$-axis and the $b$-axis. The dye molecules are stacked along the $a$-axis in a brick-wall fashion ${ }^{[2(c)]}$ with a slip angle of $c a .24^{\circ}$. This dye chromophore has an unsymmetrical intramolecular charge transfer (CT) system. Therefore, $\mathbf{1}$ has a large dipole moment in the ground state along the CT direction of the molecular plane. It should be noted that the polar molecules are aligned in the same direction in all the stacking layers. This is intriguing because molecules with a large ground state dipole moment generally align themselves to minimize electrostatic repulsion resulting in a non-polar or less-polar crystal structure. ${ }^{[21]}$ The transition dipole moment of the visible absorption was estimated to be aligned in the same direction as the ground state dipole moment. Therefore, this two-dimensional layer can be regarded as a model structure for J-aggregates, specifically the two-dimensional brick-wall structure.

\section{[Figure 4]}

The effect of the exciton interactions in this J-aggregate structure was estimated by using the extended dipole model ${ }^{[1(\mathrm{~d}), 2(\mathrm{~d}), 6]}$ on the basis of a nearest neighbour approximation. The interaction from six neighbouring dye molecules in the same layer induced a bathochromic energy shift of $991 \mathrm{~cm}^{-1}$. The contribution from two neighbouring dye molecules in the neighbouring layers was a hypsochromic energy shift of $155 \mathrm{~cm}^{-1}$. Therefore, the total from eight neighbouring molecules resulted in a bathochromic energy shift of $836 \mathrm{~cm}^{-1}$. This suggests that this solvate has an excitonic electronic nature resembling that of 
J-aggregates and this was imparted by the two-dimensional brick-wall structure, even though the transition dipole moments in the stacking layer are aligned with a small declination, which may result in negligible breaking of the symmetry limitation for light absorption based on exciton interactions. ${ }^{[2]}$

[Figure 5]

We then measured the optical properties of this solvate. The powder samples were characterized by the crystal structure of the solvate using powder X-ray diffraction. The absorption and fluorescence spectra of 1 (in toluene) and the solvate are illustrated in Figure 5. Generally it is not easy to obtain an absorption spectrum of dye crystals because dye solids have a strong extinction over the visible region. The measurements were then performed using optical waveguide spectroscopy. ${ }^{[22]}$ Dye $\mathbf{1}$ exhibits an absorption maximum around $480 \mathrm{~nm}$ in toluene with a molar extinction coefficient of $c a .2 .5 \times 10^{4}$. Its fluorescence in toluene was recognized around $556 \mathrm{~nm}$ with 21\% quantum efficiency, which was measured using quinine sulphate as a reference. The Stoke's shift is $2848 \mathrm{~cm}^{-1}$. The absorption spectrum of the solvate was found to shift drastically towards the bathochromic region. Its $\lambda$ max was around $559 \mathrm{~nm}$ with a broad shoulder around $460 \mathrm{~nm}$. The bathochromic shift of the absorption maximum from a toluene solution to the solvate was estimated to be $2944 \mathrm{~cm}^{-1}$. This spectral shift is qualitatively in agreement with the calculated bathochromic shift $\left(836 \mathrm{~cm}^{-1}\right)$ based on exciton interactions. Fluorescence of the solvate was measured by using a homemade set-up with excitation at $375 \mathrm{~nm}$. Its quantum efficiency was estimated in comparison with purified anthracene crystals. The fluorescence spectrum comprised a sharp fluorescence band around $619 \mathrm{~nm}$ and a broad component in the longer wavelength region. Its quantum efficiency is $c a .10 \%$. This sharp peak was found to decrease gradually during the measurements with a simultaneous increase of the longer wavelength component. The longer fluorescence component was also observed from the black and reddish-purple phases and thus this spectral change should be caused by the local phase transition from the solvate to these phases. This sharp fluorescence peak can be considered to reflect the excitonic band formation.

These separated two-dimensional stacking dye layers by solvent molecules are regarded as a possible structure for realizing quasi-low dimensional electronic states in a three dimensional crystalline state, i.e. 
crystals exhibit the aggregate properties. To make the distance between the adjacent brick-wall layers being larger than an effective exciton coherent length may adjust the optical properties of the solvate to those of typical J-aggregates such as sharp line-like absorption and resonance fluorescence. The stability of the solvates is also an important parameter in their practical applications.

\section{Conclusions}

A 2,3-dicyanopyrazine dye, 5-t-butyl-2,3-dicyano-6-[4-(dimethylamino)styryl]-pyrazine, was found to form a chloroform solvate which emit intense red fluorescence. Its optical property was interpreted in terms of intermolecular interactions based on two-dimensional brick-wall structure, one of the proposed structure for J-aggregates, in the crystal. The present result suggests an interesting idea for achieving J-aggregates in a three-dimensional crystalline state based on a solvate structure, although crystal engineering of such a small organic molecule is still a tough task.

\section{Acknowledgements}

We thank Dr. Eiko Yasui and Prof. Dr. Norio Takamura at Musashino University for their kind support in the dye synthesis. We also thank Dr Shinji Ishihara at Yokohama National University for the elemental analysis.

\section{References}

[1] (a) Bird GR, Norland KS, Rosenoff AE, Michaud HB. Photosensitization of silver bromide. V. Spectra and structure of sensitizing dye aggregates. Photo Sci Eng 1968; 12: 196-206; (b) The Theory of the Photographic Processes. 4th ed. James TH, editor. Macmillan Publishing, New York: 1977; (c) Möbius D. Scheibe Aggregates. Adv Mat 1995; 7: 437-444; (d) J-aggregates. Kobayashi T, editor. World Scientific Publishing, Singapore, 1996; (e) Misawa K. Self-assembled nanostructure of organic dyes

-J-aggregates-. Oyo-butsuri 2000; 69: 1401-1411 (in Japanese); (f) Würthner F, Kaiser TE, Saha-Möller CR. J-Aggregates: From Serendipitous Discovery to Supramolecular Engineering of Functional Dye 
Materials. Angew Chem Int Ed 2011; 50: 3376-3410.

[2] (a) McRae EG, Kasha M. Enhancement of Phosphorescence Ability upon Aggregation of Dye Molecules. J Chem Phys 1958; 28: 721-722; (b) Kasha M. Energy Transfer Mechanisms and the Molecular Exciton Model for Molecular Aggregates. Radiat Res 1963; 20: 55-70; (c) Czikkely V, Försterling HD, Kuhn H. Light absorption and structure of aggregates of dye molecules. Chem Phys Lett 1970; 6: 11-14; (d) Czikkely V, Försterling HD, Kuhn H. Extended dipole model for aggregates of dye molecules. Chem Phys Lett 1970; 6: 207-210; (e) Egorov VV. Theory of the J-band: From the Frenkel exciton to charge transfer. Physics Procedia 2009; 2: 223-326; (f) Spano FC, The Spectral Signatures of Frenkel Polarons in H- and J-Aggregates. Acc Chem Res 2010; 43: 429-439.

[3] Scheibe G. Die Stereoisomerie organischer Farbstoffe und ihr Zusammenhang mit Konstitution und Eigenschaften reversibel polymerer Farbstoffe. Angew Chem 1939; 42: 631-637.

[4] (a) Emerson ES, Conlin MA, Rosenoff AE, Norlamd KS, Rodriguez H, Chin D, Bird GR. The geometrical structure and absorption spectrum of a cyanine dye aggregate. J Phys Chem 1967; 71: 2396-2403; (b) Wolthaus L, Schaper A, Möbius D. Brickstone arrangement in J-aggregates on an amphiphilic merocyanine dye. Chem Phys Lett 1994; 225: 322-326; (c) Higgins DA, Barbara PF. Excitonic transitions in J-aggregates probed by near-field scanning optical microscopy. J Phys Chem 1995; 99: 3-7; (d) Higgins DA, Reid PJ, Barbara PF. Structure and Exciton Dynamics in J-Aggregates Studied by Polarization-Dependent Near-Field Scanning Optical Microscopy. J Phys Chem 1996; 100: 1174-1180; (e) Mal'tsev EI, Lypenko DA, Perelygina OM, Ivanov VI, Gribkova OL, Brusentseva MA, Vannikov AV. Molecular nanocrystals in polyaniline-based light-emitting diode structures. Protection of Metals 2008; 44: 443-446.

[5] (a) Smith DL. Structure of dyes and dye aggregates. Evidence from crystal structure analyses. Photogr Sci Eng 1974; 18: 309-322; (b) Nakatsu K, Yoshioka H, Nishigaki S. Structural Models for Low-Dimensional Photographic Dye Aggregates as Revealed by X-Ray Crystallographic Studies. Nihon Shashin Gkkai-shi (in Japanese) 1983; 46: 89-98; (c) Polymethine dyes. Tyutyulkov N, Fabian J, Mehlhorn A, Dietsand F, Tadjer A, St Kliment Ohridski University Press, Sophia; 1991. 
[6] Kato N, Yuasa K, Araki T, Hirosawa I, Sato M, Ikeda N, Iimura KI, Uesu U. Determination of a Merocyanine J-Aggregate Structure and the Significant Contribution of the Electric Dipole Interaction to the Exciton Band Wavelength. Phys Rev Lett 2005; 94: 136404.1-136404.4.

[7] (a) Kopainsky B, Kaiser W. Ultrafast transient processes of monomers, dimers, and aggregates of pseudoisocyanine chloride (PIC). Chem Phys Lett 1982; 88: 357-361; (b) Sandström V, Gillbro T, Gadonas RA, Piskarskas A. Annihilation of singlet excitons in J aggregates of pseudoisocyanine (PIC) studied by pico- $^{-}$and subpicosecond spectroscopy. J Chem Phys 1988; 89: 2754-2762; (c) Wang Y. Resonant third-order optical nonlinearity of molecular aggregates with low-dimensional excitons. J Opt Soc Am B 1991; 8: 981-985; (d) Minoshima K, Taiji M, Misawa K, Kobayashi T. Femtosecond nonlinear optical dynamics of excitons in J-aggregates. Chem Phys Lett 1994; 218: 67-72.

[8] (a) Misawa K, Ono H, Minoshima K, Kobayashi T. New model of excitonic bands and molecular arrangement of highly oriented J-aggregates in polymer films prepared by a novel method. J Lumin 1994; 60\&61: 812-815; (b) Misawa K, Machida S, Horie K, Kobayashi T. Wavelength and polarization dependence of spectral hole-burning efficiency in highly oriented J-aggregates. Chem Phys Lett 1995; 240: 210-215.

[9] (a) Malyshev VA, Glaeske H, Feller KH. Intrinsic optical bistablility of an ultrathin film consisting of oriented linear aggregates. J Chem Phys 2000; 113: 1170-1176; (b) Tatsuura S, Tian M, Furuki M, Sato Y, Pu LS, Wada O. Spin-coated Films of Squarylium Dye J-Aggregates Exhibiting Ultrafast Optical Responses. Jpn J Appl Phys 2000; 39: 4782-4785; (c) Murakami H, Morita R, Watanabe T, Asai K, Honma I, Zhou H, Yamashita M, Ishigure K, Sekigawa H. Determination of Third-Order Optical Nonlinearity Dispersion of 1-Methyl-1'-Octadecyl-2,2'-Cyanine Perchlorate Langmuir-Blodgett Films Using Electroabsorption Spectroscopy. Jpn J Appl Phys 2000; 39: 5838-5841; (d) Plekhanov AI, Gorkovenko AI, Orlova NA, Simanchuk AE, Shelkovnikov VV. A comparative study of the nonlinear optical properties of molecular J- and H-aggregates in thin films. High Energy Chem 2009; 43: 607-610.

[10] (a) Saito K. Sensitization of the Photocurrent in C60/Merocyanine J-Aggregate Heterojunction Photovoltaic Cells. Jpn J Appl Phys 1999; 38: L1140-L1142; (b) Hosokai T, Aoyama T, Kobayashi T, 
Nakao, Matsumoto S. Photovoltaic properties of bisazomethine dye thin films. Chem Phys Lett 2010; 487: 77-80.

[11] (a) Era M, Adachi C, Tsutsui T, Saito S. Double-heterostructure electroluminescent device with cyanine-dye bimolecular layer as an emitter. Chem Phys Lett 1991; 178: 488-490; (b) Era M, Adachi C, Tsutsui T, Saito S. Organic electroluminescent device with cyanine dye Langmuir-Blodgett film as an emitter. Thin Solid Films 1992; 210/211: 468-470; (c) Mal'tsev EI, Lypenko DA, Shapiro BI, Brusentseva MA, Berendyaev VI, Kotov BV, Vannikov AV. J-aggregate electroluminescence in dye doped polymer layers. Appl Phys Lett 1998; 73: 3641-3643; (d) Mal'tsev EI, Lypenko DA, Shapiro BI, Brusentseva MA, Milburn GHW, Wright J, Hendrikson A, Berendyaev VI, Kotov BV, Vannikov AV. Electroluminescence of polymer/J-aggregate composites. Appl Phys Lett 1999; 75: 1896-1898; (e) Zhang Z, Xu B, Su J, Shen L, Xie Y, Tian H. Color-tunable solid-state emission of 2,2'-biindenyl-based fluorophores. Angew Chem Int Ed 2011; 50: 11654-11657.

[12] (a) Matsumoto S, Kobayashi T, Aoyama T, Wada T. J-Aggregates in vapor deposited films of a bisazomethine dye. Chem Comm 2003; 15: 1910-1911; (b) Kim B-S, Kashibuchi D, Son Y-A, Kim S-H, Matsumoto S. Effect of phenyl ring substitution on J-aggregate formation ability of novel bisazomethine dyes in vapour-deposited films. Dyes and Pigments 2011; 90: 56-64.

[13] Kim B-S, Jindo T, Eto R, Shinohara Y, Son Y-A, Kim S-H, Matsumoto S. Effects of alkoxy substitution on the crystal structure of 2,3-bis[(E)-4-(diethylamino)-2-alkoxybenzylideneamino]fumaronitrile derivatives. CrystEngComm 2011; 13: 5374-5383.

[14] Horiguchi E, Matsumoto S, Funabiki K, Matsui M. Substituent Effect of 2,3-Dicyanopyrazine Dyes on Solid-State Fluorescence. Bull Chem Soc Jpn 2006; 79: 799-805.

[15] Kawamura Y, Sasabe H, Adachi C. Simple Accurate System for Measuring Absolute Photoluminescence Quantum Efficiency in Organic Solid-State Thin Films. Jpn J Appl Phys 2004; 43(11A): 7729-7730.

[16] Fujitu Limited, Computer aided chemical modeling package, Tokyo, Japan, 2001.

[17] Burla MC, Camalli M, Carrozzini B, Cascarano GL, Giacovazzo C, Polidori G, Spagna R. SIR 2002: 
the program. J Appl Cryst 2003; 36: 1103.

[18] Crystal structure analysis package, Rigaku and Rigaku/MSC, The Woodlands, TX, USA, 1998-2007.

[19] Matsumoto S, Yano T, Horiguchi-Babamoto E, Matsui M. Electronic States of a 2,3-Dicyanopyrazine Dye in Vacuum-deposited Films. Mol Cryst Liq Cryst 2007; 472(1): 105/[495]-112/[502].

[20] Matsuoka M. in Colorants for Non-Textile Applications. Freeman HS, Peters AT, editors. Elsevier, 2000; 339-381.

[21] (a) Molecular Crystals and Molecules. Kitaigorodsky AI, Academic Press, New York and London: 1973; (b) Molecular Aggregation. Gavezzotti A, Oxford University Press, Oxford: 2007; (c) Nonlinear Optical Properties of Organic Molecules and Crystals. vol. 1. Chemla DS, Zyss J, editors. Academic Press, New York: 1987; (d) Curtin DY, Paul IC. Chemical consequences of the polar axis in organic solid-state chemistry. Chem Rev 1981; 81: 525-541.

[22] (a) Ogawa K, Harada J, Fujiwara T, Takahashi H. UV-vis Absorption Spectra of Powdered Materials: Direct Measurements by Optical Waveguide Spectroscopy. Chem Lett 2004; 33: 1446-1447; (b) Takahashi H, Fujita K, Ohno H. Direct Visible Spectral Analysis of Solid Samples by Optical Waveguide Spectroscopy due to Adsorbed Sample Molecules after Sublimation. Chem Lett 2007; 36: 116-117. 


\section{List of Figures}

Figure 1 (a) Chemical structure of $\mathbf{1}$ and (b) molecular structure of its chloroform solvate (30\% probability level ellipsoids).

Figure 2 Photographs of dye $\mathbf{1}$ under (a) a room light and (b) a UV light.

Figure 3 The TG/DTA data for the solvate.

Figure 4 Crystal structure of the solvate.

Figure 5 UV-vis absorption (the dotted line) and fluorescence (the solid line) spectra of $\mathbf{1}$ (in toluene) and the solvate measured in an ambient condition. 